\title{
Ontogenetic change in the auditory conditioned stimulus pathway for eyeblink conditioning
}

\author{
John H. Freeman ${ }^{1}$ and Matthew M. Campolattaro \\ Department of Psychology, University of lowa, lowa City, lowa 52242, USA
}

\begin{abstract}
Two experiments examined the neural mechanisms underlying the ontogenetic emergence of auditory eyeblink conditioning. Previous studies found that the medial auditory thalamus is necessary for eyeblink conditioning with an auditory conditioned stimulus (CS) in adult rats. In experiment 1 , stimulation of the medial auditory thalamus was used as a CS in rat pups trained on postnatal days (P) 17-18, 24-25, or 31-32. All three age groups showed significant acquisition relative to unpaired controls. However, there was an age-related increase in the rate of conditioning. Experiment 2 examined the effect of inactivating the medial auditory thalamus with muscimol on auditory eyeblink conditioning in rats trained on P17-18, 24-25, or 31-32. Rat pups trained on P24-25 and P31-32, but not P17-18, showed a significant reduction in conditioned responses following muscimol infusions. The findings suggest that the thalamic contribution to auditory eyeblink conditioning continues to develop through the first postnatal month.
\end{abstract}

Developmental studies of associative learning in rats have demonstrated that conditioning emerges ontogenetically at different ages depending on the sensory modality of the conditioned stimulus (CS) and the particular conditioned response (CR) that is measured (Rudy 1992; Hunt and Campbell 1997). Auditory and visual conditioning typically develops later than conditioning with gustatory or olfactory stimuli (Rudy 1992). Within each CS sensory modality, conditioned freezing develops before conditioned changes in heart rate, which develops before potentiated startle and eyeblink conditioning (Sananes et al. 1988; Stanton et al. 1992; Hunt and Campbell 1997). Eyeblink conditioning using an auditory CS emerges as an approximately linear function of age between postnatal days (P) 17 and 24 (Stanton et al. 1992, 1998).

Recent findings indicate that the relatively late development of eyeblink conditioning may be related to maturation of sensory inputs to the cerebellum (Freeman Jr. et al. 2005; Campolattaro and Freeman 2008). The cerebellum is the site of learning and long-term memory underlying eyeblink conditioning (Thompson 2005; Ohyama et al. 2006). It receives sensory input from the unconditioned stimulus (US) through the inferior olive climbing fiber projection (Mauk et al. 1986; Steinmetz et al. 1989; Thompson 2005). The cerebellum receives sensory input from the CS through the pontine mossy fiber projection (Steinmetz et al. 1986, 1987, 1989; Knowlton and Thompson 1988; Tracy et al. 1998; Hesslow et al. 1999; Freeman Jr. and Rabinak 2004). Stimulation of mossy fiber input to the cerebellum is a sufficient CS for eyeblink conditioning in adult animals (Steinmetz et al. 1986, 1989; Tracy and Steinmetz 1998; Hesslow et al. 1999; Freeman Jr. and Rabinak 2004). It is also a sufficient CS for eyeblink conditioning in rat pups trained on P17, and as early as P12 (Freeman Jr. et al. 2005; Campolattaro and Freeman 2008). Rat pups given the mossy fiber stimulation CS on P17 condition at the same rate as rats conditioned on P24, suggesting that the mossy fiber pathway is mature enough to support cerebellar learning before conditioning can be established with an external stimulus (e.g., a tone). The sufficiency of mossy fiber stimulation as a CS for eyeblink conditioning in young rats indicates that the cerebellum is

\footnotetext{
${ }^{1}$ Corresponding author.
}

E-mail john-freeman@uiowa.edu; fax (319) 335-0191.

Article is online at http://www.learnmem.org/cgi/doi/10.1101//m.1131208. capable of learning before the pontine nuclei receive adequate sensory input. Further support for this hypothesis comes from an examination of pontine neuronal activity in developing rats (Freeman Jr. and Muckler 2003). The proportion of single units showing short-latency responses to a tone CS increased as a function of age. Moreover, there was a developmental increase in the magnitude of CS-elicited activity among units that showed tone responses. The findings of the stimulation studies and the neuronal recording study suggest that there is a developmental change in sensory input to the pontine nuclei, which limits CSrelated input to the cerebellum during eyeblink conditioning.

The pontine nuclei receive sensory input from many cortical and subcortical sources (Glickstein et al. 1972; Graybiel 1974; Kawamura 1975; Legg et al. 1989; Wells et al. 1989; Campolattaro et al. 2007). Within the auditory system, the pontine nuclei receive monosynaptic input from the auditory cortex, cochlear nuclei, inferior colliculus, and the medial nuclei of the auditory thalamus (Kawamura 1975; Steinmetz et al. 1987; Campolattaro et al. 2007). Auditory cortical input to the pontine nuclei is not necessary for acquisition or retention of eyeblink conditioning (Oakley and Russell 1972, 1977; Mauk and Thompson 1987). However, the cochlear nucleus, inferior colliculus, and medial auditory thalamic nuclei (MATN) have been shown to provide necessary and sufficient sensory input to the pontine nuclei for eyeblink conditioning in adult rabbits and rats (Steinmetz et al. 1987; Halverson and Freeman 2006; Campolattaro et al. 2007; Freeman et al. 2007; Halverson et al. 2008). The findings from studies using adult rats support a model of the auditory CS pathway that includes the medial auditory thalamus as the most proximal sensory input to the pontine nuclei (Fig. 1).

The current study was designed to determine whether developmental changes in the medial auditory thalamic input to the pontine nuclei could play a role in the ontogeny of eyeblink conditioning.

\section{Results}

Experiment 1: Stimulation of the medial auditory thalamus as a CS in developing rats

The first experiment examined whether stimulation of the medial auditory thalamus could serve as a sufficient CS for eyeblink 


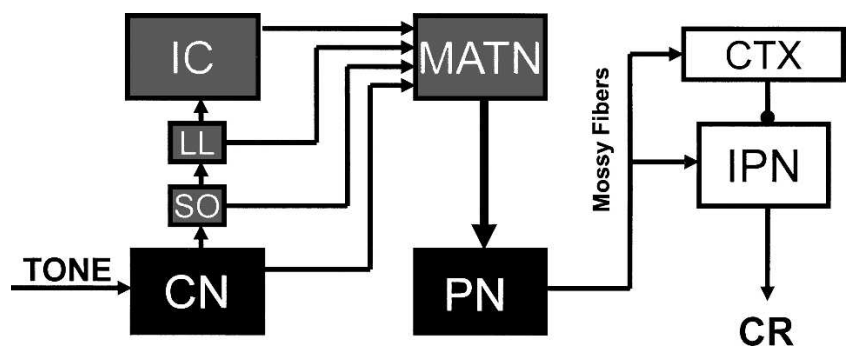

Figure 1. Diagram of the hypothesized auditory CS pathway for delay eyeblink conditioning in adult rats. Auditory CS information initially projects to the cochlear nucleus $(\mathrm{CN})$. The $\mathrm{CN}$, superior olive (SO), nucleus of the lateral leminiscus (LL), and inferior colliculus (IC) then send converging inputs to the medial auditory thalamic nuclei (MATN). Auditory information is then conveyed through a direct projection to the basal pontine nuclei $(\mathrm{PN})$. The $\mathrm{PN}$ sends mossy fiber projections into the cerebellar cortex (CTX) and interpositus nucleus (IPN). Finally, the IPN sends output for conditioned response (CR) expression.

conditioning in rats trained on P17-18, P24-25, or P31-32. A previous study found rapid acquisition of eyeblink conditioning in adult rats given medial auditory thalamic stimulation as a CS (Campolattaro et al. 2007). An age-related increase in conditioning using medial auditory thalamic stimulation as a CS would suggest that the thalamo-pontine projection is undergoing maturation that plays a role in the ontogeny of auditory eyeblink conditioning. The absence of an age-related change in conditioning using medial auditory thalamic stimulation as the CS would, on the other hand, suggest that the thalamo-pontine projection is mature early and that the development of this pathway could not account for the ontogeny of auditory eyeblink conditioning.

\section{Stimulation electrode placement}

The tips of the stimulating electrodes were positioned within 0.5 $\mathrm{mm}$ of the medial division of the medial geniculate in all of the rats in this study (Fig. 2). Moreover, the electrode placement was consistent between the three age groups. The electrode placement was also consistent with the most effective site for medial

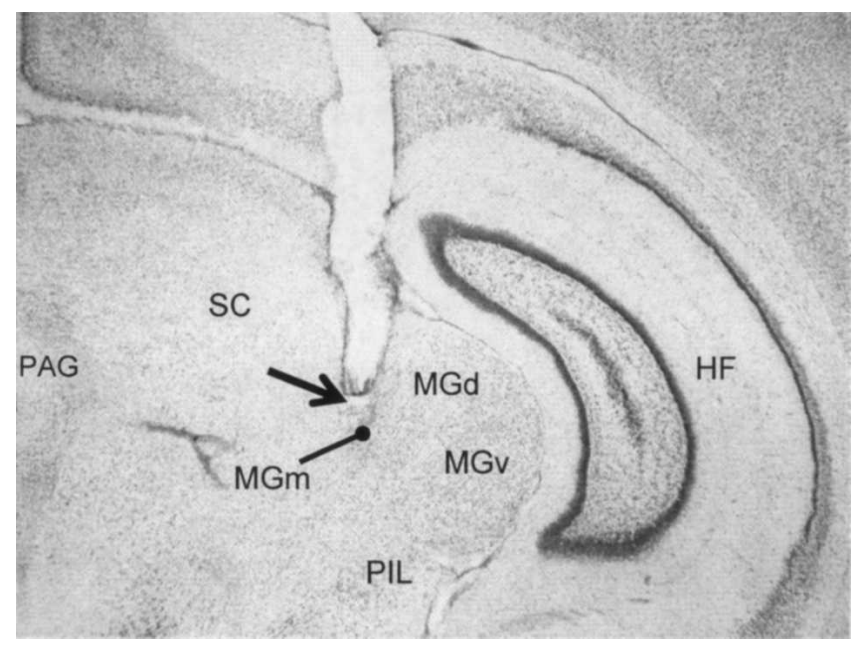

Figure 2. Digital image showing a representative electrode placement. The tip of the stimulation electrode (arrow) is within the dorsal portion of the medial division of the medial geniculate (MGm). HF indicates hippocampal formation; MGd, dorsal division of the medial geniculate; MGv, ventral division of the medial geniculate; PAG, periaqueductal gray; PIL, posterior intralaminar nucleus; and SC, intermediate superior colliculus. Magnification $=2.5 \times$. auditory thalamic stimulation in adult rats (Campolattaro et al. 2007).

\section{Eyeblink conditioning}

All age groups acquired associative eyeblink conditioning with stimulation of the MATN as the CS. Each age group (P17-18, P24-25, P31-32) showed a higher percentage of eyeblink CRs during training in the rats given paired training relative to the unpaired controls (Fig. 3). However, there was an age-related increase in the percentage of CRs among the rats given paired training (Fig. 3).

An ANOVA of the CR percentage data revealed an interaction of the age and training condition factors $\left(F_{(2,37)}=1.47\right.$, $P<0.01)$. Post-hoc tests confirmed that all of the age groups (P17-18, P24-25, P31-32) given paired conditioning showed significantly more CRs than their unpaired controls (all $P \mathrm{~s}<0.05$ ). Among the groups given paired training, the P17-18 group produced fewer CRs than the P24-25 and P31-32 groups $(P<0.05)$, and the P24-25 group produced fewer CRs than the P31-32 group $(P<0.05)$.

Conditioned response amplitude, onset latency, and peak latency were taken from CS-alone test trials in which a CR occurred to detect longer latency responses in the absence of the unconditioned response (UR). These measures of CR performance were assessed during the last three training sessions in the groups given paired training to provide a sufficient number of responses in each group for ANOVA. Conditioned response amplitude increased as a function of age, as seen in a main effect of the age factor $\left(F_{(2,20)}=7.36, P<0.005\right)$ (Fig. 4). There was also an effect of the training session factor $\left(F_{(2,40)}=4.67, P<0.02\right)$, which was due to an increase in CR amplitude between sessions 4 and 6 . No significant group effects were found for the analyses of CR onset or peak latencies, although trends toward age-related decreases were observed for both latency measures (Fig. 4).

\section{Experiment 2: Muscimol inactivation of the medial auditory thalamus during auditory eyeblink} conditioning in developing rats

The results of experiment 1 suggest that the efficacy of the thalamo-pontine pathway for auditory CSs continues developing between P24 and P31. It was, therefore, important to determine whether eyeblink conditioning that is established in rat pups with a tone CS requires the medial auditory thalamus. It was possible that the robust conditioning observed on P24-25, or

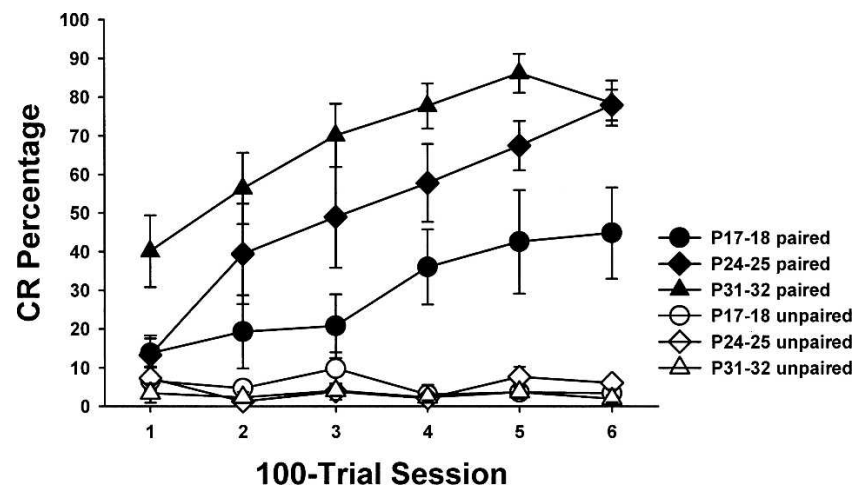

Figure 3. Medial auditory thalamic stimulation as a conditioned stimulus supports associative eyeblink conditioning in rat pups. Mean conditioned response (CR) percentage for rats given paired (black symbols) or unpaired (white symbols) training on postnatal days 17-18 (circles), 2425 (diamonds), and 31-32 (triangles) across six 100-trial sessions. Note the age-related increase in CR percentage between the groups given paired training. 


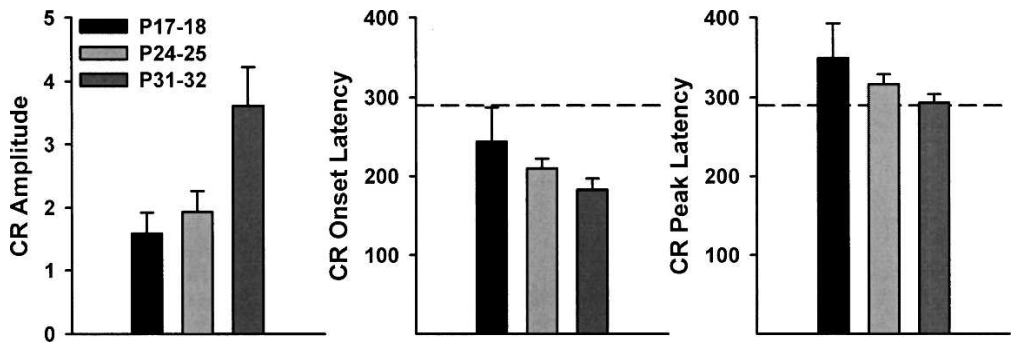

Figure 4. Age-related differences in CR amplitude (left), onset latency (middle), and peak latency (right) were evident when medial auditory thalamic stimulation was used as the conditioned stimulus. All three measures were taken from trials in which a CR occurred during the last three training sessions. Dashed lines indicate the onset time of the unconditioned stimulus.

even the modest conditioning seen on P17-18 was driven by monosynaptic projections from the cochlear nucleus and inferior colliculus to the pontine nuclei. A previous study in adult rats found that inactivation of the medial auditory thalamus with muscimol blocked acquisition and retention of auditory eyeblink conditioning (Halverson et al. 2008). The same method was used in experiment 2 to examine the effect of inactivating the medial auditory thalamus on eyeblink conditioning with a tone CS in rats conditioned on P17-18, P24-25, or P32-32.

\section{Cannula placement}

The cannula placement was very similar to the electrode placement in experiment 1 . The tips of the injection cannulae were within $0.5 \mathrm{~mm}$ of the medial division of the medial geniculate (Fig. 5). Cannula placement was consistent across the age groups and was consistent with effective cannula placements in adult rats (Halverson et al. 2008). Infusion of fluorescent muscimol in the same volume and concentration as the unlabeled muscimol resulted in a relatively discrete injection within the medial auditory thalamus (Fig. 6).

\section{Eyeblink conditioning}

Rats given eyeblink conditioning with a tone CS showed equivalent acquisition on P24-25 and P31-32, but weaker acquisition

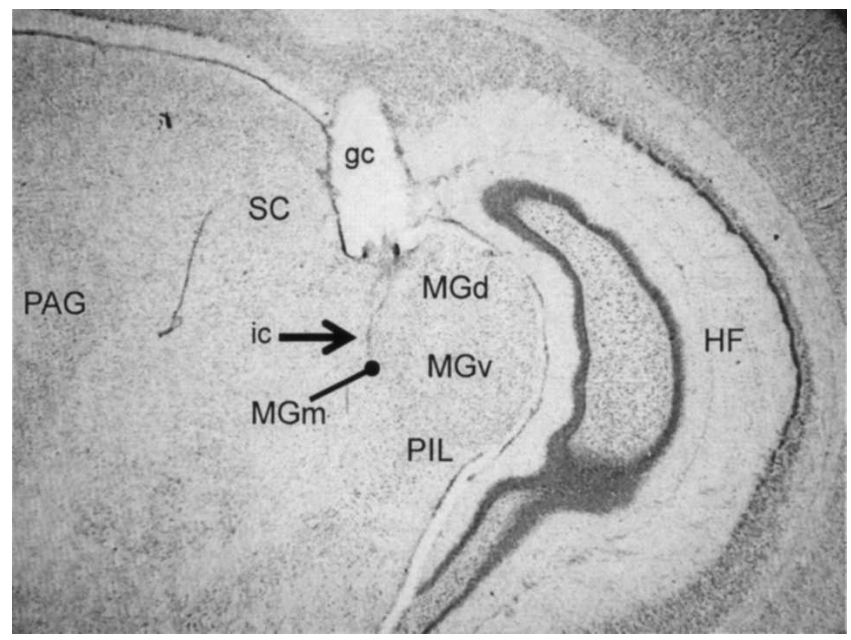

Figure 5. Digital image showing a representative cannula placement. The tip of the injection cannula (ic, arrow) is within the medial division of the medial geniculate (MGm). gc indicates guide cannula; HF, hippocampal formation; MGd, dorsal division of the medial geniculate; MGv, ventral division of the medial geniculate; PAG, periaqueductal gray; PIL, posterior intralaminar nucleus; and SC, intermediate superior colliculus. Magnification $=2.5 \times$. on P17-18 (Fig. 7). Muscimol infusion into the medial auditory thalamus resulted in a large decrement in CR percentage in the rats trained on P24-25 and P31-32, but not in the rats trained on P17-18, primarily because of a low CR percentage during the preinfusion session. During the recovery session with saline infusions, the rats trained on P24-25 and P31-32 showed complete restoration of CR percentage.

The above description of the behavioral results was supported by a within-subjects ANOVA that revealed an interaction of the age and sessions factors $\left(F_{(12,32)}=17.48, P<0.001\right)$. Post-hoc tests indicated that the CR percentage of the groups trained on P2425 or P31-32 did not differ across any of the training sessions. In contrast, the CR percentage of both of these groups was greater than the group trained on P17-18 during sessions $3-5$ and $7(P<0.05)$. The groups trained on P24-25 or P31-32 showed a significant increase in CR percentage from session 1 to sessions $3-5(P<0.05)$, whereas the increase in CR percentage across sessions in the group trained on P17-18 was not significant. During the muscimol test session (session 6), CR percentage was significantly reduced in the groups trained on P24-25 and P31-32 $(P<0.05)$, but was not reduced in the group trained on P17-18. The percentage of CRs then returned to the premuscimol levels in the groups trained on P24-25 and P31-32 during the recovery session with a saline infusion (session 7).

\section{Discussion}

Stimulation of the medial auditory thalamus was an effective CS for eyeblink conditioning in developing rats, as seen in a previous experiment that used adult rats (Campolattaro et al. 2007). The groups given paired training showed an increase in CRs across training sessions, whereas the groups given unpaired conditioning did not show a change in responding across training sessions. The increase in eyeblink CRs in the groups given paired training can therefore be attributed to associative learning. An age-related increase in associative learning was observed between groups trained on P17-18, P24-25, and P31-32. Eyeblink conditioning with a tone CS was impaired by muscimol infusion into the medial auditory thalamus in pups trained on P24-25 and P31-32.

The age-related increase in associative conditioning with medial auditory thalamus stimulation as the CS suggests that the thalamo-pontine projection (Campolattaro et al. 2007) is undergoing substantial developmental change between P17 and P31. A very surprising aspect of these data is the difference in conditioning between pups trained on P24-25 and P31-32. As seen in experiment 2, and previous studies (Freeman Jr. et al. 1995a,b), delay eyeblink conditioning with external stimuli is very similar between these age groups. The late developmental change in conditioning with thalamic stimulation suggests that the thalamo-pontine pathway continues to develop beyond P24-25. In fact, this pathway may continue to develop past P31-32, a hypothesis supported by the finding that adult rats trained with medial auditory thalamic stimulation as the CS reach asymptotic levels of conditioning within the first training session (Campolattaro et al. 2007), whereas the group conditioned on P31-32 did not reach asymptote until the third or fourth training session.

Inactivation of the medial auditory thalamus after initial acquisition produces a 70\% reduction of eyeblink CRs in adult rats (Halverson et al. 2008). A similar deficit in performance was seen in the rat pups trained on P24-25 and P31-32 in the current study. This finding indicates that the medial auditory thalamus is a necessary component of the auditory CS pathway in develop- 


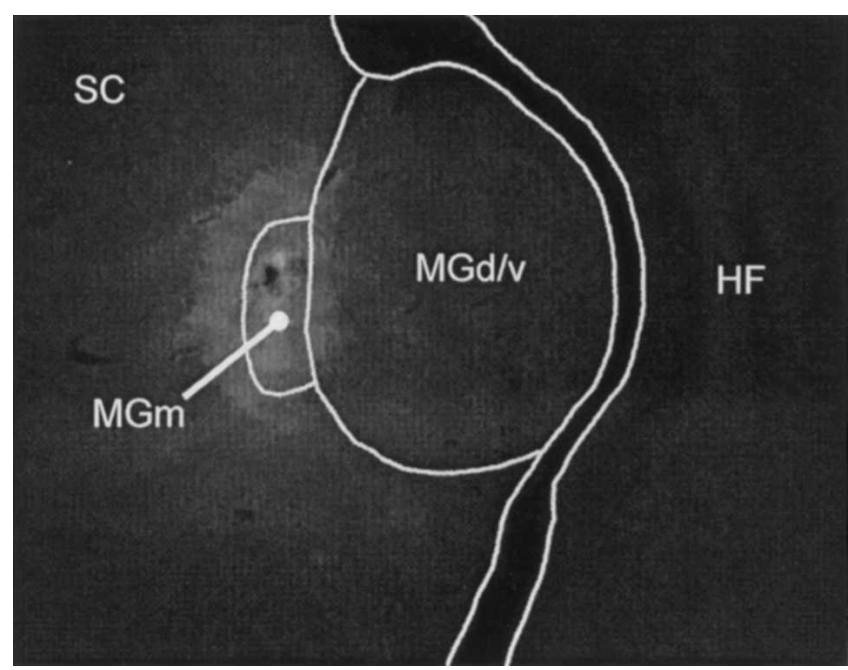

Figure 6. A digital image of fluorescent muscimol $(0.2 \mu \mathrm{L}, 2 \mathrm{nmol})$ within the medial auditory thalamus showing labeling within the medial division of the medial geniculate $(\mathrm{MGm})$ but very little labeling within the dorsal or ventral medial geniculate (MGd/v). HF indicates hippocampal formation; SC, superior colliculus. Magnification $=2.5 \times$.

ing rats. The severe deficit in CR performance with inactivation of the auditory thalamus also suggests that the monosynaptic projections of the cochlear nucleus and inferior colliculus to the pontine nuclei do not play an essential role in conditioning with a tone CS in rat pups. The essential components of the auditory CS pathway in eyeblink conditioning area are, therefore, the same in adult and developing rats (Halverson and Freeman 2006; Halverson et al. 2008).

Acquisition of eyeblink conditioning was considerably faster with medial auditory thalamic stimulation as a CS relative to conditioning with a tone CS (cf. CR percentages for sessions $1-5$ in Figs. 3 and 7). The group trained on P17-18 showed very little conditioning with the tone CS but showed an increase in CR percentage with the thalamic stimulation CS by session 5 . Pups trained on P24-25 or P31-32 also showed stronger conditioning with thalamic stimulation relative to conditioning with

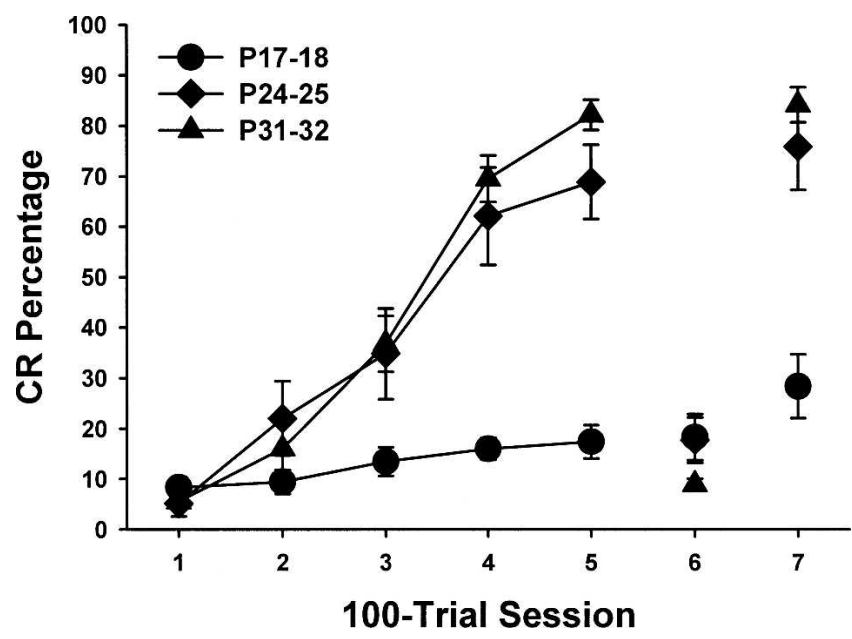

Figure 7. Mean conditioned response (CR) percentage for rats given paired training on postnatal days 17-18 (circles), 24-25 (diamonds), and 31-32 (triangles) across seven 100-trial sessions. Muscimol was infused into the medial auditory thalamus before session 6 . Note the age-related increase in CR percentage during sessions 1-5 and the reduction in CR percentage in the groups trained on P24-25 and P31-32. the tone CS. In contrast to the group trained on P17-18, however, the enhancement of conditioning with medial auditory thalamic stimulation was seen early in training in the older pups. The group trained on P31-32 showed the greatest enhancement of conditioning with thalamic stimulation, which was most evident during the first session (Fig. 3). The enhanced performance of rat pups and adult rats (Campolattaro et al. 2007) given medial auditory thalamic stimulation as a CS indicates that activation of the thalamo-pontine pathway is a highly effective stimulus. This finding provides further evidence that the monosynaptic projection from the medial auditory thalamus to the pontine nuclei is a key component of the necessary and sufficient auditory CS pathway for eyeblink conditioning (Campolattaro et al. 2007; Halverson et al. 2008).

The specific nature of the developmental change in the thalamo-pontine auditory pathway is not evident from the current findings. Developmental changes in the axonal projection may occur and are under investigation. It is also possible that the axonal projection precedes maturation of excitatory synaptic transmission in this pathway. Developmental changes in neuronal excitability within the medial auditory thalamus or pontine nuclei are also possible mechanisms underlying the developmental changes in the thalamo-pontine pathway. Whatever the specific ontogenetic mechanism is underlying the development of the thalamo-pontine pathway, it plays a substantial role in the ontogeny of eyeblink conditioning. Developmental changes in auditory thalamic input to the pontine nuclei would also influence sensory input into the cerebellum. Accordingly, weaker mossy fiber input to the cerebellar cortex and deep nuclei from the CS pathway in younger animals would result in less synaptic plasticity and weaker conditioning. Current experiments are examining the aforementioned ontogenetic mechanisms that could underlie the development of the thalamo-pontine pathway.

The findings of this study suggest that the ontogeny of eyeblink conditioning is driven by the development of the CS pathway to the cerebellum between P17 and P31. Previous studies using pontine stimulation as a CS indicate that the mossy fiber projection is sufficiently mature to support eyeblink conditioning as early as P12 (Freeman Jr. et al. 2005; Campolattaro and Freeman 2008). This finding combined with the current results suggests that the site of developmental change in the auditory CS pathway is the thalamo-pontine projection. It is important to note, however, that rat pups are capable of associative learning with an auditory stimulus as early as P14 with appetitive conditioning (Hyson and Rudy 1984) and P16 with aversive (fear) conditioning (Hunt and Campbell 1997). Thus, the thalamo-pontine pathway appears to be late developing relative to the auditory pathways that are necessary for appetitive and fear conditioning. An important developmental issue for future study is to determine when the fear conditioning circuitry begins to interact with the eyeblink conditioning circuitry to facilitate acquisition, as seen in adult animals (Lee and Kim 2004; Blankenship et al. 2005).

\section{Materials and Methods \\ Experiment 1}

\section{Subjects}

Forty three Long-Evans rat pups at different three ages, P17-18 (paired $n=13)$, P24-25 ( $n=17)$, and P31-32 $(n=13)$, from 22 litters were used as subjects. No more than one same-sex littermate was assigned to a group. 


\section{Surgery}

Surgery occurred $1 \mathrm{~d}$ before the beginning of training. Rat pups were anesthetized with i.p. injections of ketamine $(100 \mathrm{mg} / \mathrm{kg})$, xylazine $(5 \mathrm{mg} / \mathrm{kg})$, and atropine $(0.8 \mathrm{mg} / \mathrm{kg})$. Differential (EMG) electrodes were implanted in the left upper eyelid, and a ground electrode was connected to one of two skull hooks. The second skull hook was secured to the skull slightly anterior to lambda. A bipolar stimulating electrode was then implanted into right medial auditory thalamus, targeting the medial division of the medial geniculate $(\mathrm{MGm})$. The stereotaxic coordinates for the $\mathrm{MGm}$, with the skull level, were taken from lambda (P16/P23/P30: $+2.5 /$ $+2.5 /+2.4$ anterior, $-2.5 /-2.6 /-2.7$ medial-lateral, $-5.3 /-5.6 /$ -5.8 dorsal-ventral). Once the electrode was in place, it was secured with dental acrylic covering the entire length of the electrode above the skull surface, including the plastic connector.

\section{Conditioning apparatus}

The conditioning apparatus consisted of a small-animal sound attenuation chamber with a small-animal operant chamber inside (BRS/LVE). The rats were kept in the operant chamber during conditioning. Cables with connectors for the EMG, US, and CS electrodes were attached to a commutator. The electrode leads from the rat's head stage were connected to peripheral equipment and a desktop computer. Computer software controlled the delivery of stimuli and recording of eyelid EMG activity (JSA Designs). The US was delivered through a stimulus isolator (World Precision Instruments). EMG activity was recorded differentially, filtered (500-5000 Hz), amplified $(2000 \times)$, and integrated (time constant, $20 \mathrm{msec}$ ). MGN stimulation was triggered through a programmable stimulator (Master 8, A.M.P.I.), which controlled signal input to a stimulus isolator (World Precision Instruments) that delivered the electrical stimulation.

\section{MATN stimulation}

Electrical stimulation of the medial auditory thalamus functioned as the CS, which was administered in a $200-\mathrm{Hz}$ train of 0.1 -msec biphasic pulses for $300 \mathrm{msec}$. The stimulation threshold for the CS was found before training by setting the stimulating current to elicit an observable behavioral response and then decreasing the current in 5- $\mu \mathrm{A}$ increments until no movement was detected. Observable movements included but were not limited to eyeblinks, orienting responses, ear movements, and head movements. The maximum stimulation level was $100 \mu \mathrm{A}$ (range, 30-100 $\mu \mathrm{A}$ ). The most typical behavioral response elicited by stimulation was a discrete movement of the left ear. The mean stimulation intensities were $92.5 \mu \mathrm{A}$ on P17-18, $87.5 \mu \mathrm{A}$ on P2425 , and $58.1 \mu \mathrm{A}$ on P31-32.

\section{Conditioning}

Rats were given six paired or unpaired training sessions. The paired training sessions consisted of 100 trials; each with 90 trials of the stimulation CS paired with the shock US (10 msec, 1.0-2.0 $\mathrm{mA}$ ) and 10 stimulation CS-alone trials, occurring on every 10 th trial. The CS-alone trials were included to assess behavioral responses (integrated EMG activity) uncontaminated by URs. The interstimulus interval for paired trials was $290 \mathrm{msec}$. Trials were separated by an intertrial interval that averaged $30 \mathrm{sec}$. Unpaired training included explicitly unpaired presentations of the stimulation CS and US, with an intertrial interval that averaged $15 \mathrm{sec}$. Behavioral data were examined from computer records of EMG activity. CRs were defined as responses that crossed a threshold of 0.4 arbitrary units above the baseline activity during the CS period, but at least $80 \mathrm{msec}$ after CS onset, to avoid contamination of the CR measures by the startle (alpha) response. The amplitude, onset latency, and peak latency of the CR were measured on CS-alone trials in which a CR occurred.

\section{Histology}

After training was completed, the rats were euthanized with a lethal injection of sodium pentobarbital $(90 \mathrm{mg} / \mathrm{kg})$ and transcardially perfused with $0.1 \mathrm{M}$ buffered saline, followed by a $10 \%$ buffered formalin solution. The brains were post-fixed in forma- lin for $2 \mathrm{~d}$ and then put in a solution of 30\% sucrose before sectioning. The brains were sectioned at $50 \mu \mathrm{m}$ with a sliding microtome. Sections were then stained with thionin. The bipolar stimulating electrode location was determined by examining a series of sections.

\section{Experiment 2}

\section{Subjects}

Twenty-five Long-Evans rat pups at different three ages, P17-18 $(n=9), \mathrm{P} 24-25(n=8)$, and P31-32 $(n=8)$, from 11 litters were used as subjects. No more than one same-sex littermate was assigned to a group.

\section{Surgery}

The surgical procedure was the same as in experiment 1, except that isoflurane $(1 \%-3 \%)$ was used for anesthesia and a cannula was implanted in the medial auditory thalamus instead of a stimulating electrode. A 27-gauge (thin walled) guide cannula was implanted $0.5 \mathrm{~mm}$ dorsal to the injection site. A stylet made from a 33-gauge cannula was placed in the guide cannula until the muscimol test and the recovery session.

\section{Muscimol infusion}

Before the muscimol infusion, the stylet was removed from the guide cannula and replaced with a 33-gauge infusion cannula that extended $0.5 \mathrm{~mm}$ beyond the guide cannula. The infusion cannula was connected to polyethylene tubing (PE 10; 110-120 $\mathrm{cm}$ ), which was connected to a $10-\mu \mathrm{L}$ gas tight syringe (Hamilton). The syringe was placed in an infusion pump (Harvard Apparatus), and $0.2 \mu \mathrm{L}$ of muscimol $(2 \mathrm{nmol}, \mathrm{pH} 7.4)$ or saline was infused over $2 \mathrm{~min}$ at a rate of $6 \mu \mathrm{L} / \mathrm{h} 40 \mathrm{~min}$ before the test session. The infusion cannula remained in place for the duration of the test session.

\section{Apparatus, conditioning, and histology}

The apparatus, conditioning, and histology procedures were identical to those used in experiment 1 , except that a tone CS $(2.0 \mathrm{kHz}, 85 \mathrm{~dB})$ was used instead of medial auditory thalamus stimulation.

\section{Acknowledgments}

This work was supported by NINDS Grant NS038890 to J.H.F. We thank Stephanie D. Bajo for technical assistance.

\section{References}

Blankenship, M.R., Huckfeldt, R., Steinmetz, J.J., and Steinmetz, J.E. 2005. The effects of amygdala lesions on hippocampal activity and classical eyeblink conditioning in rats. Brain Res. 1035: 120-130.

Campolattaro, M.M. and Freeman, J.H. 2008. Eyeblink conditioning in 12-day-old rats using pontine stimulation as the conditioned stimulus. Proc. Natl. Acad. Sci. 105: 8120-8123.

Campolattaro, M.M., Halverson, H.E., and Freeman, J.H. 2007. Medial auditory thalamic stimulation as a conditioned stimulus for eyeblink conditioning in rats. Learn. Mem. 14: 152-159.

Freeman Jr., J.H. and Muckler, A.S. 2003. Developmental changes in eyeblink conditioning and neuronal activity in the pontine nuclei. Learn. Mem. 10: 337-345.

Freeman Jr., J.H. and Rabinak, C.A. 2004. Eyeblink conditioning in rats using pontine stimulation as a conditioned stimulus. Integr. Physiol. Behav. Sci. 39: 180-191.

Freeman Jr., J.H., Barone Jr., S., and Stanton, M.E. 1995a. Disruption of cerebellar maturation by an antimitotic agent impairs the ontogeny of eyeblink conditioning in rats. J. Neurosci. 15: 7301-7314.

Freeman Jr., J.H., Carter, C.S., and Stanton, M.E. 1995b. Early cerebellar lesions impair eyeblink conditioning in developing rats: Differential effects of unilateral lesions on postnatal day 10 or 20 . Behav. Neurosci. 109: 893-902.

Freeman Jr., J.H., Rabinak, C.A., and Campolattaro, M.M. 2005. Pontine stimulation overcomes developmental limitations in the neural mechanisms of eyeblink conditioning. Learn. Mem. 12: 255-259.

Freeman, J.H., Halverson, H.E., and Hubbard, E.M. 2007. Inferior colliculus lesions impair eyeblink conditioning in rats. Learn. Mem. 14: $842-846$. 
Glickstein, M., Stein, J., and King, R.A. 1972. Visual input to the pontine nuclei. Science 178: 1110-1111.

Graybiel, A.M. 1974. Visuo-cerebellar and cerebello-visual connections involving the ventral lateral geniculate nucleus. Exp. Brain Res. 20: $303-306$.

Halverson, H.E. and Freeman, J.H. 2006. Medial auditory thalamic nuclei are necessary for eyeblink conditioning. Behav. Neurosci. 120: $880-887$.

Halverson, H.E., Poremba, A., and Freeman, J.H. 2008. Medial auditory thalamus inactivation prevents acquisition and retention of eyeblink conditioning. Learn. Mem. 15: 532-538.

Hesslow, G., Svensson, P., and Ivarsson, M. 1999. Learned movements elicited by direct stimulation of cerebellar mossy fiber afferents. Neuron 24: 179-185.

Hunt, P.S. and Campbell, B.A. 1997. Developmental dissociation of the components of conditioned fear. In Learning, motivation, and cognition: The functional behaviorism of Robert C. Bolles (eds. M.E. Bouton and M.S. Fanselow), pp. 53-74. American Psychological Association, Washington, DC.

Hyson, R.L. and Rudy, J.W. 1984. Ontogenesis of learning: II. Variation in the rat's reflexive and learned responses to acoustic stimulation. Dev. Psychobiol. 17: 263-283.

Kawamura, K. 1975. The pontine projection from the inferior colliculus in the cat. An experimental anatomical study. Brain Res. 95: 309-322.

Knowlton, B.J. and Thompson, R.F. 1988. Microinjections of local anesthetic into the pontine nuclei reduce the amplitude of the classically conditioned eyelid response. Physiol. Behav. 43: 855-857.

Lee, T. and Kim, J.J. 2004. Differential effects of cerebellar, amygdalar, and hippocampal lesions on classical eyeblink conditioning in rats. J. Neurosci. 24: 3242-3250.

Legg, C.R., Mercier, B., and Glickstein, M. 1989. Corticopontine projection in the rat: The distribution of labelled cortical cells after large injections of horseradish peroxidase in the pontine nuclei. $J$. Comp. Neurol. 286: 427-441.

Mauk, M.D. and Thompson, R.F. 1987. Retention of classically conditioned eyelid responses following acute decerebration. Brain Res. 403: 89-95.

Mauk, M.D., Steinmetz, J.E., and Thompson, R.F. 1986. Classical conditioning using stimulation of the inferior olive as the unconditioned stimulus. Proc. Natl. Acad. Sci. 83: 5349-5353.

Oakley, D.A. and Russell, I.S. 1972. Neocortical lesions and Pavlovian conditioning. Physiol. Behav. 8: 915-926.

Oakley, D.A. and Russell, I.S. 1977. Subcortical storage of Pavlovian conditioning in the rabbit. Physiol. Behav. 18: 931-937.

Ohyama, T., Nores, W.L., Medina, J.F., Riusech, F.A., and Mauk, M.D. 2006. Learning-induced plasticity in deep cerebellar nucleus. $J$. Neurosci. 26: 12656-12663.

Rudy, J.W. 1992. Development of learning: From elemental to configural associative networks. Adv. Infancy Res. 7: 247-289.

Sananes, C.B., Gaddy, J.R., and Campbell, B.A. 1988. Ontogeny of conditioned heart rate to an olfactory stimulus. Dev. Psychobiol. 21: 117-133.

Stanton, M.E., Freeman Jr., J.H., and Skelton, R.W. 1992. Eyeblink conditioning in the developing rat. Behav. Neurosci. 106: 657-665.

Stanton, M.E., Fox, G.D., and Carter, C.S. 1998. Ontogeny of the conditioned eyeblink response in rats: Acquisition or expression? Neuropharmacology 37: 623-632.

Steinmetz, J.E., Rosen, D.J., Chapman, P.F., Lavond, D.G., and Thompson, R.F. 1986. Classical conditioning of the rabbit eyelid response with a mossy-fiber stimulation CS: I. Pontine nuclei and middle cerebellar peduncle stimulation. Behav. Neurosci. 100: $878-887$.

Steinmetz, J.E., Logan, C.G., Rosen, D.J., Thompson, J.K., Lavond, D.G., and Thompson, R.F. 1987. Initial localization of the acoustic conditioned stimulus projection system to the cerebellum essential for classical eyelid conditioning. Proc. Natl. Acad. Sci. 84: 3531-3535.

Steinmetz, J.E., Lavond, D.G., and Thompson, R.F. 1989. Classical conditioning in rabbits using pontine nucleus stimulation as a conditioned stimulus and inferior olive stimulation as an unconditioned stimulus. Synapse 3: 225-233.

Thompson, R.F. 2005. In search of memory traces. Annu. Rev. Psychol. 56: $1-23$.

Tracy, J.A. and Steinmetz, J.E. 1998. Purkinje cell responses to pontine stimulation CS during rabbit eyeblink conditioning. Physiol. Behav. 65: $381-386$.

Tracy, J.A., Thompson, J.K., Krupa, D.J., and Thompson, R.F. 1998. Evidence of plasticity in the pontocerebellar conditioned stimulus pathway during classical conditioning of the eyeblink response in the rabbit. Behav. Neurosci. 112: 267-285.

Wells, G.R., Hardiman, M.J., and Yeo, C.H. 1989. Visual projections to the pontine nuclei in the rabbit: Orthograde and retrograde tracing studies with WGA-HRP. J. Comp. Neurol. 279: 629-652.

Received June 30, 2008; accepted in revised form August 28, 2008. 


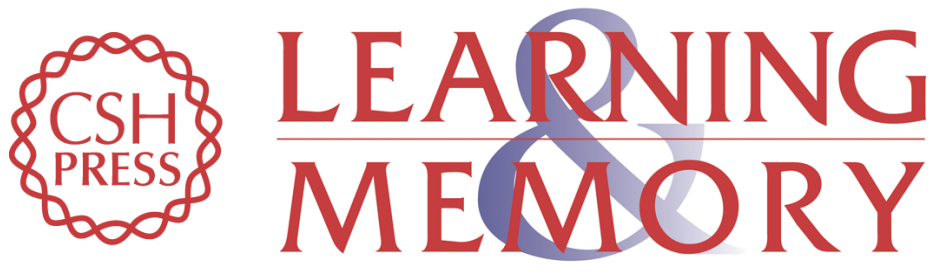

\section{Ontogenetic change in the auditory conditioned stimulus pathway for eyeblink conditioning}

John H. Freeman and Matthew M. Campolattaro

Learn. Mem. 2008, 15:

Access the most recent version at doi:10.1101/lm.1131208

References This article cites 35 articles, 12 of which can be accessed free at:

http://learnmem.cshlp.org/content/15/11/823.full.html\#ref-list-1

License

Email Alerting Receive free email alerts when new articles cite this article - sign up in the box at the Service top right corner of the article or click here. 